\title{
Acquired Bilateral Cataracts
}

National Cancer Institute

\section{Source}

National Cancer Institute. Acquired Bilateral Cataracts. NCI Thesaurus. Code C101191.

Cataracts in both eyes that result from the aging process, an injury, or as a manifestation of a systemic disorder. 